\title{
BEYOND TIME-FREQUENCY ANALYSIS: ENERGY DENSITIES IN ONE AND MANY DIMENSIONS
}

\author{
Richard G. Baraniuk \\ Department of Electrical and Computer Engineering \\ Rice University \\ P.O. Box 1982, Houston, TX 77251-1892, USA
}

\begin{abstract}
Given a unitary operator $\mathcal{A}$ representing a physical quantity of interest, we employ concepts from group representation theory to define two natural signal energy densities for $\mathcal{A}$. The first is invariant to $\mathcal{A}$ and proves useful when the effect of $\mathcal{A}$ is to be ignored; the second is covariant to $\mathcal{A}$ and measures the " $A$ " content of signals. The construction is quite general and is also easily extended to the multi-operator case, which generalizes previously derived joint densities such as the timefrequency and time-scale distributions.
\end{abstract}

\section{INTRODUCTION}

Time-frequency representations, which indicate the energy content of signals simultaneously in both time and frequency, have proven indispensable for the study of the nonstationary signals appearing in many applications, including speech, radar, biological, and transient signal analysis and processing [1]. Most time-frequency representations (the spectrogram and the Wigner distribution are two examples) are based on the time and frequency shift operators and, consequently, perform well in applications where time and frequency shifts are fundamental. In other problems, such as sonar and image processing, the concept of scale (compression/dilation) is more relevant than frequency shift; hence joint time-scale representations have been developed $[1,2]$, the most popular of which is certainly the wavelet transform.

While time-frequency and time-scale representations are the natural analysis and processing tools for large classes of signals, they are not perfectly matched to all signals, just as time shifts, frequency shifts, and scale changes are not the fundamental transformations appearing in all applications. For these different classes of signals, joint representations based on concepts other than time, frequency, and scale must be developed. Some progress has been made in this direction $[3,4]$; however, at present there is no adequate theory for generating joint representations in the general case.

In this paper, we take a step towards this goal by formulating a theory for joint distributions of broad classes of physical quantities that include, but are not limited to, time, frequency, and scale. Specifically, given unitary operators $A$ and $B$ representing two physical quantities of interest, we will derive classes of distributions that measure the joint " $\mathcal{A}-\mathcal{B}$ " energy content of signals.

Following a background section containing some results from functional analysis and group theory, we will proceed in steps of increasing complexity, beginning with onedimensional transforms that measure the $\mathcal{A}$ content of signals and culminating in the construction of $\mathcal{A}-\mathcal{B}$ distributions. While our solution to this problem is quite general, its construction is also strikingly simple, owing to the powerful group theoretic arguments employed.

\section{PRELIMINARIES}

The approach of this paper is to associate physical quantities with parameterized unitary operators on a Hilbert function space $H[3,4]$. In signal processing applications, $H$ is typically one of the $L^{2}\left(G, d \mu_{G}\right)$ spaces, with $G$ the set of function indices and $d \mu_{G}$ a measure on that set. These spaces have inner product $\langle g, h\rangle=\int_{G} g(x) h^{*}(x) d \mu_{G}(x)$ for $g, h \in L^{2}\left(G, d \mu_{G}\right)$ and norm $\|h\|^{2}=\langle h, h\rangle$.

Assuming that the function index $x \in G$ represents a time coordinate, we define the time and frequency operators on $L^{2}(\mathbb{R}, d x)$ as the unitary ${ }^{1}$ time shift $\left(\mathcal{T}_{t} g\right)(x)=g(x-t)$ and unitary frequency shift $\left(\mathcal{F}_{f} g\right)(x)=e^{j 2 \pi f x} g(x)$, respectively. We define as scale operators the unitary dilations $\left(\mathcal{D}_{d}^{\prime} g\right)(x)=$ $d^{-1 / 2} g(x / d), d>0$, on $L^{2}\left(\mathbb{R}_{+}, d x\right)$ (one-sided signals) and $\left(\mathcal{D}_{d} g\right)(x)=g(x / d), d>0$, on $L^{2}\left(\mathbb{R}_{+}, d x / x\right)$. If $x \in G$ does not represent a time coordinate, then the interpretations of $\mathcal{T}, \mathcal{F}$, and $\mathcal{D}$ must be adjusted accordingly. For example, if $x$ represents frequency, then $\mathcal{T}$ corresponds to a frequency shift and $\mathcal{F}$ corresponds to a time shift.

Given a parameterized linear operator $\mathcal{A}_{a}$ on $H$, solution of the eigenequation

$$
\left(\mathcal{A}_{a} \mathbf{e}_{\alpha}^{\mathcal{A}}\right)(x)=\lambda_{a, \alpha}^{\mathcal{A}} \mathbf{e}_{\alpha}^{\mathcal{A}}(x)
$$

yields the eigenfunctions $\left\{e_{\alpha}^{A}(x)\right\}$ and the eigenvalues $\left\{\lambda_{a, \alpha}^{A}\right\}$ of $\mathcal{A}_{a}$, both of which are indexed by the parameter $\alpha$. The eigenvalues and eigenfunctions of the time and frequency operators on $L^{2}(\mathbb{R}, d x)$ are easily shown to be

$$
\lambda_{t, f}^{\mathcal{T}}=\lambda_{f, t}^{\mathcal{F}}=e^{-j 2 \pi f t}, \quad \mathbf{e}_{f}^{\mathcal{T}}(x)=e^{j 2 \pi x}, \quad \mathbf{e}_{t}^{\mathcal{F}}(x)=\delta(x+t),
$$

while the eigenvalues and eigenfunctions of the scale operators are given by

$$
\lambda_{d, \eta}^{\mathcal{D}}=e^{-j 2 \pi \eta \log d}, \quad \mathrm{e}_{\eta}^{D}(x)=e^{j 2 \pi \eta \log x}
$$

on $L^{2}\left(\mathbb{R}_{+}, d x / x\right)$ and by $\lambda_{d, \eta}^{\mathcal{D}^{\prime}}=\lambda_{d, \eta}^{\mathcal{D}}, \mathbf{e}_{\eta}^{\mathcal{D}^{\prime}}(x)=x^{-1 / 2} \mathbf{e}_{\eta}^{\mathcal{D}}(x)$ on $L^{2}\left(\mathbb{R}_{+}, d x\right)$.

If $\mathcal{A}_{a}$ is unitary, then $\left|\lambda_{a, \alpha}^{A}\right|=1$, and the eigenfunctions form a complete orthonormal set in $H$. The expansion onto these eigenfunctions then yields another operator that we will refer to as the $\mathcal{A}$-Fourier transform $\mathrm{F}_{\mathcal{A}}$

$$
\left(\mathbf{F}_{\mathcal{A}} s\right)(\alpha)=\left\langle s, \mathbf{e}_{\alpha}^{A}\right\rangle=\int_{G} s(x) \mathbf{e}_{\alpha}^{A *}(x) d \mu_{G}(x) .
$$

\footnotetext{
${ }^{1}$ A unitary operator $\mathcal{U}$ is a linear transformation from a Hilbert space $H$ back onto itself that preserves energy; that is, $\mid \mathcal{U g}^{2}=$ $|g|^{2}$ for all $g \in H$. Unitary operators also preserve inner products (isometry); that is, $\langle\mathcal{U} g, \mathcal{U} h\rangle=\langle g, h\rangle$ for any unitary $\mathcal{U}$ and for all $g, h \in H$. A norm-preserving operator mapping one Hilbert space onto another is termed an isometric isomorphism.
} 
Being an isometric isomorphism, $\mathbf{F}_{\mathcal{A}}$ is also invertible. To continue the example from above, $\hat{F}_{T}$ is the usual Fourier transform (unitary on $\left.L^{2}(\mathbb{R}, d x)\right),\left(F_{\mathcal{F}} s\right)(t)=s(-t)$, and $F_{\mathcal{D}}$ is a Mellin transform $[3,4]$

$$
\left(\mathbf{F}_{\mathcal{D}} s\right)(\eta)=\left\langle s, \mathbf{e}_{\eta}^{\mathcal{D}}\right\rangle=\int_{0}^{\infty} s(x) e^{-j 2 \pi \eta \log x} \frac{d x}{x}
$$

shown here for $s \in L^{2}\left(\mathbb{R}_{+}, d x / x\right)$.

Because a unitary operator maps $H$ back onto itself in a manner that preserves exactly its structure - it does not change the distances or angles between vectors - unitary operators can be interpreted as basis transformations, prompting the following definition of operators that are equivalent modulo a change of basis $[\mathbf{x}, \mathbf{y}]$ :

Deflnition 1 Two operators $\mathcal{A}$ and $B$ are unitarily equivalent if we can write $B=\mathcal{U}^{-1} \mathcal{A} U$ with $\mathcal{U}$ a unitary transformation.

The time and frequency operators are unitarily equivalent, since $\mathcal{F}_{k}=\mathbf{F}_{T}^{-1} \mathcal{T}_{k} \mathbf{F}_{T}$. The time operator is not equivalent to either of the scale operators $\mathcal{D}_{d}$ or $\mathcal{D}_{d}^{\prime}$, however.

The underlying structure of a unitary operator is unchanged by a unitary equivalence transformation. For example, given operators $\mathcal{A}_{k}$ and $B_{k}=U^{-1} \mathcal{A}_{k} U$, it is straightforward to show that

$$
\lambda_{k, \beta}^{\mathcal{B}}=\lambda_{k, \beta}^{\mathcal{A}}, \quad \mathbf{e}_{\beta}^{\mathcal{B}}=U^{-1} \mathbf{e}_{\beta}^{\mathcal{A}}, \quad \mathbf{F}_{\mathcal{B}}=\mathbf{F}_{\mathcal{A}} U .
$$

A powerful tool for studying invariants of any kind is group theory. ${ }^{2}$ Each parameterized operator that we use to represent a physical quantity can be interpreted as a unitary representation of some group. The particular group and group operation corresponding to a representation $\mathcal{A}_{a}$ are given by the domain of the parameter $a$ and its behavior when $\mathcal{A}_{a}$ is concatenated with itself: $A_{x} A_{y}=A_{x \bullet y}$. It follows immediately that all unitarily equivalent operators are representations of the same group, since if $B=\mathcal{U}^{-1} \mathcal{A} \mathcal{U}$, then

$B_{x \bullet y}=B_{x} B_{y}=\left(\mathcal{U}^{-1} \mathcal{A}_{x} U\right)\left(\mathcal{U}^{-1} \mathcal{A}_{y} U\right)=\mathcal{U}^{-1} \mathcal{A}_{x \bullet y} U$.

Thus, the group concept partitions parameterized unitary operators into mutually exclusive equivalence classes.

While we started out this section referring to the time, frequency, and scale operators as "parameterized unitary operators," we can now see them for that they really are: the time and frequency operators are both representations of the group $(\mathbb{R},+)$ of real numbers with addition as the group operation, whereas the scale operators are representations of the group $\left(\mathbb{R}_{+}, *\right)$ of positive real numbers with multiplication as the group operation. Although, for the sake of brevity we will consider only these two groups for examples in this paper, note that all results are valid for arbitrary locally compact abelian (LCA) groups [5].

Given an LCA group $G$ with group operation $\bullet$, the simplest possible representation of $G$ on $L^{2}\left(G, d \mu_{G}\right)$ is the group translation operator $\tau^{G}$, defined as

$$
\left(\tau_{r}^{G} s\right)(x)=s\left(x \bullet r^{-1}\right), \quad s \in L^{2}\left(G, d \mu_{G}\right), \quad x, r \in G .
$$

Translation is unitary if the measure $d \mu_{G}$ is chosen to be

\footnotetext{
${ }^{2}$ A set $G$ with binary operation $\bullet$ is called a group if: 1) $G$ is closed under $\bullet$; that is, $x \bullet y \in G \forall x, y \in G$; 2) the operation is associative; that is $x \bullet(y \bullet z)=(x \bullet y) \bullet z \forall x, y, z \in G$; 3) there exists an identity element $\theta \in G$ such that $x \bullet \theta=\theta \bullet x=x \forall x \in G$; 4) for each $x \in G$ there exists an inverse element $x^{-1}$ such that $x^{-1} \bullet x=x \bullet x^{-1}=\theta$. A group $G$ is abelian (commutative) if $x \bullet y=y \bullet x \forall x, y \in G[5]$.
}

invariant to $\tau^{G}$; that is, if

$$
\int_{G}\left(\tau_{r}^{G} s\right)(x) d \mu_{G}(x)=\int_{G} s(x) d \mu_{G}(x), \quad \forall r \in G .
$$

The invariant measure can be regarded as the natural measure for the group $G$, singling out the space $L^{2}\left(G, d \mu_{G}\right)$ as the natural space on which the operators unitarily equivalent to $\tau^{G}$ act. We will assume from this point onwards that the invariant measure is always employed in $L^{2}\left(G, d \mu_{G}\right)$. Continuing the examples, for the group $(\mathbb{R},+)$, the invariant measure is $d \mu_{G}(x)=d x$ and the group translation operator is $\mathcal{T}_{r}$, while for the group $\left(\mathbb{R}_{+}, *\right)$, the invariant measure is $d \mu_{G}(x)=d x / x$ and the group translation operator is $\mathcal{D}_{r}$.

The fact that all unitary representations of a group $G$ share common eigenvalues (see (2)) inspires the group Fourier transform $\mathbf{F}_{G}[5]$

$$
\left(\mathbf{F}_{G} s\right)(\alpha)=\int_{G} s(x) \lambda_{x, a}^{G *} d \mu_{G}(x)
$$

where $\lambda^{G}$ denotes the common eigenvalues. The transformation $F_{G}$ is an isomorphic isomorphism from the space $L^{2}\left(G, d \mu_{G}\right)$ onto the space $L^{2}\left(\Gamma, d \mu_{\Gamma}\right)$ based on the dual group $\Gamma$ of $G{ }^{3}$ Consequently, the inverse group transform of a function $S(\alpha) \in L^{2}\left(\Gamma, d \mu_{\Gamma}\right)$ is defined similarly, as

$$
\left(\mathbf{F}_{G}^{-1} S\right)(x)=\int_{\Gamma} S(\alpha) \lambda_{x, \alpha}^{G} d \mu_{\Gamma}(\alpha) .
$$

On $L^{2}\left(G, d \mu_{G}\right)$ (using the invariant measure), the group Fourier transform coincides with the $\tau^{G}$-Fourier transform; therefore, the $\mathcal{A}$-Fourier transforms of all operators unitarily equivalent to $\tau^{G}$ can be obtained as $F_{A}=F_{G} U$, where $\mathcal{A}_{k}=\mathcal{U}^{-1} \tau_{k}^{G} \mathcal{U}$. To complete the examples for this section, note that the $(\mathbb{R},+)$ group Fourier transform is the usual Fourier transform mapping $L^{2}(\mathbb{R}, d x)$ onto $L^{2}(\mathbb{R}, d x)$. The $\left(\mathbb{R}_{+}, *\right)$ group Fourier transform is the Mellin transform mapping $L^{2}\left(\mathbb{R}_{+}, d x / x\right)$ onto $L^{2}(\mathbb{R}, d x)$ (the dual group is $(\mathbb{R},+)$ ).

The results of this paper spring immediately from the characterization of physical quantities (time, frequency, scale, and so on) as group objects, specifically, as unitary representations of groups on certain Hilbert signal spaces. We will now apply this powerful mathematical machinery to the problem at hand: energy densities in one and many dimensions.

\section{ENERGY DENSITIES}

In this section, we define two natural transforms for a unitary representation $\mathcal{A}$ of an LCA group. The first is invariant to $\mathcal{A}$, while the second truly measures the " $\mathcal{A}$ " content of signals. It is useful to keep in mind as models the operators $\mathcal{T}$ and $\mathcal{F}$ and the transformations $|s(t)|^{2}$ and $\left|F_{T} s\right|^{2}$, which indicate the time and frequency energy content of a time signal $s$, respectively. Note that while the results of this section are completely general, when interpreting them we will assume that the signal $s(x)$ has been expressed in the "time domain."

Invariant Energy Densities. Squaring the $\mathcal{A}$-Fourier transform yields an energy density that is invariant to the operator $\mathcal{A}$

$$
\left|\left(\mathbf{F}_{\mathcal{A}} \mathcal{A}_{\mathfrak{a}} s\right)(\alpha)\right|^{2}=\left|\left(\mathbf{F}_{\mathcal{A}} s\right)(\alpha)\right|^{2}
$$

\footnotetext{
${ }^{3}$ The second parameter $\alpha$ of $\lambda_{x, \alpha}^{G}$ belongs to the dual group $\Gamma$. We will generally use greek letters to denote elements of $\Gamma$. A notable exception is the parameter $f$ of the $\mathcal{F}_{f}$, which lies in both
} $G=(\mathbb{R},+)$ and $\Gamma=G$ 
Thus, we will refer to $\left|F_{\mathcal{A}} s\right|^{2}$ as the $\mathcal{A}$-invariant energy density $(\mathcal{A}$-IED). It is extremely important to note that the $\mathcal{A}$ IED does not indicate the $\mathcal{A}$ content of the signal $s$, precisely because of this invariance. In fact, the $\mathcal{A}$-IED is the transform of choice when the action of $\mathcal{A}$ is to be ignored!

As examples, recall that the $\mathcal{T}$-Fourier transform reduces to the usual Fourier transform, which indicates not time but frequency content. Similarly, the $\mathcal{D}$-Fourier (Mellin) transform (1) indicates not scale but some sort of "logarithmic chirp" content.

Covariant Energy Densities. Although it is clear that the energy density indicating the $\mathcal{A}$ content of a signal must change when $\mathcal{A}$ is applied to the signal, it is unclear what type of change should occur.

Definition $2 A$ transformation $\Theta$ is $\mathcal{Z}$-covariant to an operator $\mathcal{A}$ if $\Theta \mathcal{A}_{k}=\mathcal{Z}_{k} \Theta$.

The essence of $\mathcal{Z}$-covariance is that the operator $\mathcal{Z}$ describes the effect of "pulling" $\mathcal{A}$ out through the transformation $\Theta$. It follows directly from the definition that if $\mathcal{Z}$ covariance is desired in an $\mathcal{A}$-content energy density, then the density is given by $|\Theta s|^{2}$. Note that $\mathcal{Z}$ cannot be arbitrary, since $\mathcal{A}$ and $\mathcal{Z}$ are unitarily equivalent, $\mathcal{A}_{k}=\Theta^{-1} \mathcal{Z}_{k} \Theta$, and, hence, are representations of the same group $G$.

Since the natural choice for $\Theta$ should lead to the simplest possible covariance, a group-theoretic argument suggests we choose $\mathcal{Z}=\tau^{G}$, the translation operator of the group G. Therefore, we define the $\mathcal{A}$-covariant energy density $(\mathcal{A}-$ CED) as $\left|\Theta_{A} s\right|^{2}$, where $\mathcal{A}_{k}=\Theta_{A}^{-1} \tau_{k}^{G} \Theta_{\mathcal{A}}$. An illuminating interpretation of $\Theta_{A}$ is as a coordinate transformation from "time" coordinates to " $\mathcal{A}$ " coordinates, where the action of $\mathcal{A}$ is simpler than any other unitary operator based on the group $G$.

Examples. While the $\mathcal{A}$-IED does not indicate the $\mathcal{A}$ content of signals, the $\mathcal{A}-\mathrm{CED}$ does.

For the group $G=(\mathbb{R},+)$ acting on $L^{2}(\mathbb{R}, d x), \tau_{k}^{G}=\mathcal{T}_{k}$. The time and frequency operators produce the expected results: the $\mathcal{T}$-IED is the square of the usual Fourier transform $\left|\left(\mathbf{F}_{\mathcal{T}} s\right)(f)\right|^{2}$ and the $\mathcal{T}$-CED is $|s(t)|^{2}$, while for $\mathcal{F}$ these densities are reversed. Another interesting unitary representation of $G$ is the chirp modulation operator $\left(\mathcal{C}_{c} s\right)(x)=$

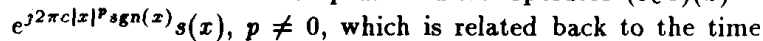
operator by $\mathcal{C}_{c}=\Theta_{\mathcal{C}}^{-1} \tau_{c}^{G} \Theta_{\mathcal{C}}$ with

$$
\left(\Theta_{\mathcal{C}} s\right)(c)=\int_{-\infty}^{\infty} s(x) e^{-j 2 \pi c|x|^{p s g n}(x)}|x|^{(p-1) / 2}|p|^{1 / 2} d x .
$$

Since the $\mathcal{C}-\operatorname{CED}\left|\left(\Theta_{\mathcal{C}} s\right)(c)\right|^{2}$ indicates the "chirp" content of signals, it has been called the chirp transform [4]. The $\mathcal{C}$-IED is given by $\left|\left(F_{\mathcal{C} s}\right)(\gamma)\right|^{2}=|p|^{-1}|\gamma|^{(1-p) / p}\left|s\left(|\gamma|^{1 / p} \operatorname{sgn}(\gamma)\right)\right|^{2}$. This transform demonstrates the nonuniqueness of IEDs, since the density $|s(\gamma)|^{2}$ is also invariant under $\mathcal{C}$.

For the group $G=\left(\mathbb{R}_{+}, *\right)$ acting on $L^{2}\left(\mathbb{R}_{+}, d x / x\right)$, $\tau_{k}^{G}=\mathcal{D}_{k}$. Thus, the $\mathcal{D}$-CED - the true scale indicating transform - is $|s(d)|^{2}, d>0$. This result may be surprising (and perhaps a little disappointing), but it is also correct, consistent, and reasonable [6]. First, this density has the correct covariance property: $\left|\left(\mathcal{D}_{k} s\right)(d)\right|^{2}=|s(d / k)|^{2}$. Second, this density indicates the scale content of the signal $s$ : just as the $\mathcal{T}$-CED $|s(t)|^{2}$ indicates the amount of group translation $\left(\tau^{G}=\mathcal{T}\right.$ in the additive group) required to bring the signal energy at the point $t$ to the identity element $\theta=0$ of that group, the $\mathcal{D}$-IED $|s(d)|^{2}$ indicates the amount of group translation $\left(\tau^{G}=\mathcal{D}\right.$ in the multiplicative group) required to bring the signal energy at the point $d$ to the identity element $\theta=1$ of that group. Third, since scale is a ratio - "this thing is twice as big as that thing" - the features on which the scale content of a signal can be judged exist in the time domain, and so no elaborate coordinate transformations are necessary. An enlightening interpretation of the $\mathcal{D}$-CED is provided by considering the index $d$ of $s(d)$ as a spatial variable in an imaging system, with $|s(d)|^{2}$ the distribution of an object along the image axis. In this case, $d$ represents a "zoom" parameter, and the spread of the $\mathcal{D}$-CED indicates the amount of focus change required to successively bring all of the object into focus at an image plane at $d=1$.

\section{JOINT ENERGY DENSITIES}

In the previous section, we defined two energy densities that are natural for any unitary representation operator $\mathcal{A}$ of an LCA group. The $\mathcal{A}$-IED is invariant to $\mathcal{A}$, while the $\mathcal{A}$ CED is covariant to $\mathcal{A}$ (and thus measures the $\mathcal{A}$ content in signals). In many applications, these densities and their linear equivalents will be more than adequate for characterizing, analyzing, and processing signals. One obvious example of an IED/CED is the square of the Fourier transform, which has found a multitude of applications.

However, for more complicated signals, several physical quantities may be meaningful simultaneously, and joint densities based on several operators are required. Time-frequency representations, for example, were developed for problems where both the time shift $\mathcal{T}$ and frequency shift $\mathcal{F}$ are important.

General Method. A joint distribution of two operators $\mathcal{A}$ and $B$ can be defined as the energy density function $\left(\mathcal{P}_{s}\right)(u, v)$ whose marginal distributions $\mathcal{M}_{\mathcal{A}}$ and $\mathcal{M}_{\mathcal{B}}$, given by

$$
\begin{aligned}
& \int\left(\mathcal{P}_{s}\right)(u, v) d \mu_{B}(v)=\left|\left(\mathcal{M}_{A} s\right)(u)\right|^{2} \\
& \int\left(\mathcal{P}_{s}\right)(u, v) d \mu_{A}(u)=\left|\left(\mathcal{M}_{B} s\right)(v)\right|^{2},
\end{aligned}
$$

are energy densities for $\mathcal{A}$ and $\boldsymbol{B}^{4}$ Natural possibilities for $\mathcal{M}_{A}$ and $\mathcal{M}_{B}$ are the IEDs and CEDs for $\mathcal{A}$ and $B$. If CEDs are chosen for both $\mathcal{A}$ and $B$ (one of the four options), then $\left(\mathcal{P}_{s}\right)(u, v)$ can be interpreted as indicating the joint $\mathcal{A}-\mathcal{B}$ content of the signal $s$.

The basis for the general method lies in the pioneering work of Cohen $[1,3]$, who developed a characteristic function approach to energy densities for the time and frequency operators. The present work generalizes his approach to covariances based on groups other than $(\mathbb{R},+)$ and introduces the choice of IED or CED marginals. For the moment, we assume that both operators $\mathcal{A}$ and $B$ arise from the same group $G$. The construction proceeds as follows:

Step 1. Choose either IED or CED marginals for $\mathcal{A}$ and $B$. For an $\mathcal{A}$-IED marginal, set $\overline{\mathcal{A}}_{a}=\mathcal{A}_{a}$, while for an $\mathcal{A}$-CED marginal, set $\overline{\mathcal{A}}_{\alpha}=\Theta_{A}^{-1} \Lambda_{\alpha}^{G} \Theta_{\mathcal{A}}$, where $\left(\Lambda_{\alpha}^{G} s\right)(x)=\lambda_{x, \alpha}^{G} s(x)$. Note that the parameter $a$ lies in $G$, while $\alpha$ lies in the dual group $\Gamma$. Form $\overline{\mathcal{B}}$ in the same manner. To illustrate, we will work towards $\mathcal{A}$-CED and $B$-IED marginals in the following.

Step 2. Form the characteristic function

$$
\left(\mathcal{Q}_{s}\right)(\alpha, b)=\phi(\alpha, b)\left\langle s, \overline{\mathcal{A}}_{\alpha} \overline{\mathcal{B}}_{b} s\right\rangle,
$$

where the inner product is taken in $L^{2}\left(G, d \mu_{G}\right)$. The ordering of $\overline{\mathcal{A}}$ and $\overline{\mathcal{B}}$ is arbitrary; distributions arising from all possible

\footnotetext{
${ }^{4}$ While joint distribution classes can also be derived using co-
} variance arguments [2], we will not follow this approach here. 
orderings can be obtained from a single ordering by an appropriate choice of the kernel function $\phi$ [3]. A symmetrical ordering leads to Wigner-like distributions. Note that when $\mathcal{A}$-IED and $B$-IED marginals are desired $(\overline{\mathcal{A}}=\mathcal{A}$ and $\bar{B}=B)$, $\mathcal{Q} s$ is an ambiguity function for $\mathcal{A}$ and $B$.

Step 3. Compute the $\mathcal{A}-\boldsymbol{B}$ distribution as the double group Fourier transform of $\mathcal{Q} s$. For IED marginals, use the forward transform; for CED marginals, use the inverse transform:

$$
\left(\mathcal{P}_{s}\right)(a, \beta)=\mathbf{F}_{G, \alpha \rightarrow a}^{-1} \mathbf{F}_{G, b \rightarrow \beta} \phi(\alpha, b)\left\langle s, \overline{\mathcal{A}}_{\alpha} \overline{\mathcal{B}}_{b} s\right\rangle .
$$

The marginal properties of $\mathcal{P}_{s}$ follow directly from the properties of the group Fourier transform, which parallel those of the usual Fourier transform [5]. We begin with the $\mathcal{A}$-CED marginal. Setting $\overline{\mathcal{A}}$ and $\overline{\boldsymbol{B}}$ for the correct marginals and integrating with respect to $d \mu_{\Gamma}(\beta)$ yields ${ }^{5}$

$$
\begin{aligned}
& \int_{\Gamma}(\mathcal{P} s)(a, \beta) d \mu_{\Gamma}(\beta)= \\
& \quad \mathbf{F}_{G_{, \alpha \rightarrow a}^{-1}} \int_{G}\left\langle s, \overline{\mathcal{A}}_{\alpha} B_{b} s\right\rangle\left[\int_{\Gamma} \lambda_{b, \beta}^{G} d \mu_{\Gamma}(\beta)\right] d \mu_{G}(b) .
\end{aligned}
$$

Formally, the term in brackets equals the Dirac delta function $\delta(\beta)$; hence, the integration over $b$ sifts the value $b=\theta$ into $\mathcal{B}$. Since $B_{\theta}=\mathcal{I}$, the identity operator, the $\mathcal{A}$ marginal of $\mathcal{P}_{s}$ equals

$$
\begin{aligned}
& \mathbf{F}_{G, \alpha \rightarrow a}^{-1}\left\langle s, \overline{\mathcal{A}}_{\alpha} s\right\rangle=\mathbf{F}_{G, \alpha \rightarrow a}^{-1}\left\langle\Theta_{\mathcal{A}} s, \Lambda_{\alpha}^{G} \Theta_{\mathcal{A}} s\right\rangle \\
& =\int_{G}\left|\left(\Theta_{\mathcal{A}} s\right)(u)\right|^{2}\left[\int_{\Gamma} \lambda_{u, \alpha}^{G *} \lambda_{a, \alpha}^{G} d \mu_{\Gamma}(\alpha)\right] d \mu_{G}(u) \\
& =\int_{G}\left|\left(\Theta_{\mathcal{A}} s\right)(u)\right|^{2}\left[\int_{\Gamma} \lambda_{a u^{-1, \alpha}}^{G} d \mu_{\Gamma}(\alpha)\right] d \mu_{G}(u) \\
& =\left|\left(\Theta_{\mathcal{A}} s\right)(a)\right|^{2},
\end{aligned}
$$

The $\mathcal{B}$-IED marginal follows similarly. Integrating $\mathcal{P}_{s}$ with respect to $d \mu_{G}(a)(a \in G)$ yields (skipping the disappearance of $\overline{\mathcal{A}}$, which occurs as above for $\mathcal{B}$ )

$$
\begin{aligned}
\mathbf{F}_{G, b \rightarrow \beta}\left\langle s, \mathcal{B}_{b} s\right\rangle=\mathbf{F}_{G, b \rightarrow \beta}\left\langle\Theta_{\mathcal{B}} s, \tau_{b}^{G} \Theta_{\mathcal{B}} s\right\rangle \\
=\int_{G}\left(\Theta_{\mathcal{B}} s\right)(u)\left[\int_{G}\left(\Theta_{\mathcal{B}} s\right)^{*}\left(u \bullet b^{-1}\right) \lambda_{b, \beta}^{G} d \mu_{G}(b)\right] d \mu_{G}(u) \\
=\int_{G}\left(\Theta_{\mathcal{B}} s\right)(u)\left[\int_{G}\left(\Theta_{\mathcal{B}} s\right)^{*}(v) \lambda_{u \bullet v^{-1}, \mathcal{B}}^{G} d \mu_{G}(v)\right] d \mu_{G}(u) \\
=\left[\int_{G}\left(\Theta_{\mathcal{B}} s\right)(u) \lambda_{u, \beta}^{G *} d \mu_{G}(u)\right]\left[\int_{G}\left(\Theta_{\mathcal{B}} s\right)^{*}(v) \lambda_{v, \beta}^{G} d \mu_{G}(v)\right] \\
=\left|\left(F_{G} \Theta_{\mathcal{B}} s\right)(\beta)\right|^{2}=\left|\left(F_{\mathcal{B}} s\right)(\beta)\right|^{2} .
\end{aligned}
$$

Examples and Extensions.

Time and Frequency. Cohen's class of time-frequency distributions $[1,3]$ is recovered by setting $\mathcal{A}=\mathcal{T}, \mathcal{B}=\mathcal{F}$ and selecting CED marginals for both operators. A symmetry exists in this case: the CED for one operator is the IED for the other. The class of $\mathcal{A}-\boldsymbol{B}$ distributions sharing this attractive property was characterized in [4] and contains distributions of all operator pairs $\mathcal{A}, B$ such that $\mathcal{A}=\mathcal{U}^{-1} \mathcal{T} U$ and $B=\mathcal{U}^{-1} \mathcal{F} U$ simultaneously.

Time and Chirp. We obtain a class of time and chirp distributions by setting $\mathcal{A}=\mathcal{T}$ and $\mathcal{B}=\mathcal{C}$, the chirp modulation operator from Section 3. For CED

\footnotetext{
${ }^{5}$ Since $\beta$ is a member of the dual group $\Gamma$, the measure $d \mu_{\Gamma}$ must be employed in (4).
}

marginals, the prescription specifies $\overline{\mathcal{A}}=\mathcal{F}$ and $\left(\overline{\mathcal{B}}_{b s}\right)(x)=$ $s\left(m_{p}\left(m_{1 / p}(x)-b\right)\right)|x|^{(1-p) / 2 p}\left|m_{1 / p}(x)-b\right|^{(p-1) / 2}$, where $m_{p}(u)=|u|^{p} \operatorname{sgn}(u)$. A symmetrical combination of these operators in the characteristic function (6) yields the Wignerlike time-chirp distribution

$$
\left(\mathcal{P}_{s}\right)(t, c)=\int_{-\infty}^{\infty}\left(\bar{B}_{-b / 2} s\right)(t)\left(\bar{B}_{b / 2} s\right)^{*}(t) e^{j 2 \pi b c} d b
$$

having as marginals $|s(t)|^{2}$ and $\left|\left(\Theta_{c} s\right)(c)\right|^{2}$ from (3).

Time and Scale. Distributions of time and scale cannot be computed directly using (7), since $\mathcal{A}$ and $B$ are assumed there to be representations of the same group $G$. However, using the fact that all 1-d LCA groups are isomorphic (equivalent) [5], we can obtain distributions of operators representing different 1-d groups via a coordinate transformation. For example, the operator $\mathcal{T}$ is equivalent to the exponentiated scale operator $\mathcal{D}_{e^{k}}^{\prime}$ on $L^{2}\left(\mathbb{R}_{+}, d x\right)$, since $\mathcal{D}_{e^{k}}^{\prime}=U_{\text {hyp }}^{-1} \mathcal{T}_{k} \mathcal{U}_{\text {hyp }}$ with $U_{\text {hyp }}$ the following isometric isomorphism

$$
\left(U_{\mathrm{hyp}} s\right)(x)=e^{x / 2} s\left(e^{x}\right) .
$$

Applying the above formulas for $\mathcal{T}$-CED vs. $\mathcal{D}$-IED distributions (the $\mathcal{T}-C E D$ vs. $\mathcal{D}$-CED distributions are trivial) yields the time-scale distributions of Marinovic and Altes [3], which have $|s(t)|^{2}$ and $\left|\left(\mathbf{F}_{\mathcal{D}^{\prime}} s\right)(\eta)\right|^{2}$ as marginals.

\section{CONCLUSIONS}

Viewing invariant and covariant signal energy densities from a group theory perspective has proven illuminating. These simple concepts are central for studying both single- and multioperator energy distributions.

While the present approach to joint distributions is a modification of that of Cohen [3], our construction differs from his in two important respects. First, by working directly with unitary operators, rather than their Hermetian counterparts, a more direct derivation results. Second, the general method provides the added flexibility of choice of any combination of IED or CED marginals. In fact, it was the inability of the formulations in [3] to manufacture a true $\mathcal{T}-\mathrm{CED}$ vs. $\mathcal{D}-\mathrm{CED}$ distribution that elicited the general approach.

Finally, note that the above formulation is easily extended to distributions of more than two operators simply by appending extra terms to the characteristic function (6).

\section{REFERENCES}

1. L. Cohen, "Time-Frequency Distributions - A Review," Proc. IEEE, vol. 77, no. 7, 1989, pp. 941-981.

2. O. Rioul and P. Flandrin, "Time-Scale Energy Distributions: A General Class Extending Wavelet Transforms," IEEE Trans. Signal Proc., vol. 40, no. 7, 1992, pp. 17461757.

3. L. Cohen, "Generalized Phase Space Distribution Functions," J. Math. Phys., vol. 7, pp. 781-786, 1966; "A General Approach to Obtaining Joint Representations in Signal Analysis and an Application to Scale," Proc. SPIE 1566, 1991; "The Scale Representation," IEEE Trans. Signal Proc., vol. 41, no. 12, 1993.

4. R. Baraniuk and D. Jones, "Warped Wavelet Bases: Unitary Equivalence and Signal Processing," ICASSP 93, vol. III, pp. 320-323; "Unitary Equivalence: A New Twist on Signal Processing," preprint.

5. W. Rudin, "Fourier Analysis on Groups," Wiley, 1962.

6. R. Baraniuk, "A Signal Transform Covariant to Scale Changes," Electronics Letters, vol. 29, no. 19, 1993. 